\title{
No Cardiac Valve Stenosis
}

National Cancer Institute

\section{Source}

National Cancer Institute. No Cardiac Valve Stenosis. NCI Thesaurus. Code C99992.

The cardiac valve orifice is not abnormally narrow. (ACC) 